\title{
A Comparison among Different Machine Learning Pretest Approaches to Predict Stress-Induced Ischemia at PET/CT Myocardial Perfusion Imaging
}

\author{
Rosario Megna $\left(\mathbb{D},{ }^{1}\right.$ Mario Petretta $\left(\mathbb{D},{ }^{2}\right.$ Roberta Assante, ${ }^{3}$ Emilia Zampella $\left(\mathbb{D},{ }^{3}\right.$ \\ Carmela Nappi $\mathbb{D}^{3},{ }^{3}$ Valeria Gaudieri $\mathbb{D}^{3},{ }^{3}$ Teresa Mannarino, ${ }^{3}$ Adriana D'Antonio, ${ }^{3}$ \\ Roberta Green $\left(\mathbb{D},{ }^{3}\right.$ Valeria Cantoni $\mathbb{D}^{3},{ }^{3}$ Parthiban Arumugam, ${ }^{4}$ Wanda Acampa ${ }^{\mathbb{D}},{ }^{1,3}$ \\ and Alberto Cuocolo ${ }^{3}{ }^{3}$ \\ ${ }^{1}$ Institute of Biostructure and Bioimaging, National Council of Research, Naples, Italy \\ ${ }^{2}$ IRCCS-SDN, Naples, Italy \\ ${ }^{3}$ Department of Advanced Biomedical Sciences, University Federico II, Naples, Italy \\ ${ }^{4}$ Department of Nuclear Medicine, Central Manchester Foundation Trust, Manchester, UK \\ Correspondence should be addressed to Rosario Megna; rosario.megna@ibb.cnr.it
}

Received 30 July 2021; Revised 29 October 2021; Accepted 15 November 2021; Published 27 November 2021

Academic Editor: Huiling Chen

Copyright (c) 2021 Rosario Megna et al. This is an open access article distributed under the Creative Commons Attribution License, which permits unrestricted use, distribution, and reproduction in any medium, provided the original work is properly cited.

\begin{abstract}
Traditional approach for predicting coronary artery disease (CAD) is based on demographic data, symptoms such as chest pain and dyspnea, and comorbidity related to cardiovascular diseases. Usually, these variables are analyzed by logistic regression to quantifying their relationship with the outcome; nevertheless, their predictive value is limited. In the present study, we aimed to investigate the value of different machine learning (ML) techniques for the evaluation of suspected CAD; having as gold standard, the presence of stress-induced ischemia by ${ }^{82} \mathrm{Rb}$ positron emission tomography/computed tomography (PET/CT) myocardial perfusion imaging (MPI) ML was chosen on their clinical use and on the fact that they are representative of different classes of algorithms, such as deterministic (Support vector machine and Naïve Bayes), adaptive (ADA and AdaBoost), and decision tree (Random Forest, rpart, and XGBoost). The study population included 2503 consecutive patients, who underwent MPI for suspected CAD. To testing ML performances, data were split randomly into two parts: training/test (80\%) and validation (20\%). For training/test, we applied a 5-fold cross-validation, repeated 2 times. With this subset, we performed the tuning of free parameters for each algorithm. For all metrics, the best performance in training/test was observed for AdaBoost. The Naïve Bayes ML resulted to be more efficient in validation approach. The logistic and rpart algorithms showed similar metric values for the training/test and validation approaches. These results are encouraging and indicate that the ML algorithms can improve the evaluation of pretest probability of stress-induced myocardial ischemia.
\end{abstract}

\section{Introduction}

Artificial intelligence has assumed a consolidated role in numerous fields and also in the healthcare and research and development. Machine learning (ML), an application of artificial intelligence that refers to computational algorithms designed to learn from experience, has been used successfully for diagnosis, prognosis, and drug development
[1-4]. Among the recommendations for ML implementation in clinical research, there is data normalization, feature selection, parameter tuning, and independent validation [5, $6]$.

In the field of cardiology, the search for methods for obtaining reliable pretests probability of disease has been underway for some time [7]. These tools should assist the physician in making decisions about referring patients for 
TABle 1: Clinical characteristics of cohort according to MPI outcome.

\begin{tabular}{|c|c|c|c|}
\hline & $\begin{array}{c}\text { Normal } \\
(n=2002)\end{array}$ & $\begin{array}{l}\text { Ischemic } \\
(n=501)\end{array}$ & $\begin{array}{c}P \\
\text { value }\end{array}$ \\
\hline Age, $n(\%)$ & & & $<0.001$ \\
\hline$<55$ & 777 (39) & $84(17)$ & \\
\hline $55-65$ & $603(30)$ & $146(29)$ & \\
\hline$>65$ & $622(31)$ & $271(54)$ & \\
\hline Male gender, $n(\%)$ & $881(44)$ & $334(67)$ & $<0.001$ \\
\hline $\begin{array}{l}\text { Body mass index } \geq 30, n \\
\text { (\%) }\end{array}$ & $1024(51)$ & $258(52)$ & 0.93 \\
\hline Chest pain, $n(\%)$ & & & $<0.001$ \\
\hline Typical & $678(34)$ & $114(23)$ & \\
\hline Atypical & $256(13)$ & $87(17)$ & \\
\hline Noncardiac* & $1068(53)$ & $300(60)$ & \\
\hline Diabetes, $n(\%)$ & $479(24)$ & $187(37)$ & $<0.001$ \\
\hline Dyspnea, $n(\%)$ & $446(22)$ & $139(28)$ & $<0.05$ \\
\hline $\begin{array}{l}\text { Family history of CAD, } \\
n(\%)\end{array}$ & $945(47)$ & $199(40)$ & $<0.005$ \\
\hline Hypertension, $n(\%)$ & $1361(68)$ & $401(80)$ & $<0.005$ \\
\hline Hyperlipidemia, $n$ (\%) & $1210(60)$ & $343(69)$ & $<0.005$ \\
\hline Smoking, $n(\%)$ & $557(28)$ & $144(29)$ & 0.72 \\
\hline $\begin{array}{l}\text { Diagnostic question, } n \\
(\%)^{\S}\end{array}$ & & & $<0.001$ \\
\hline Diagnostic evaluation & $1642(82)$ & $370(74)$ & \\
\hline Presurgery evaluation & $360(18)$ & $131(26)$ & \\
\hline
\end{tabular}

${ }^{*}$ Considering noncardiac patients as the reference. ${ }^{\circledR}$ Considering diagnostic evaluation patients as the reference.

examination. Usually, for the prediction of coronary artery disease (CAD), traditional risk factors, such as age, gender, chest pain, and comorbidity related to cardiovascular diseases, such as hypertension, diabetes, and hyperlipidemia, are considered. These variables are analyzed by logistic regression to quantifying their relationship with the outcome of the exam and obtaining predictions for new patients [8-11]. However, the models obtained by these studies do not show a great performance, probably due to the declining prevalence of CAD and because the evaluation for CAD has shifted to older patients, more women, and more patients with atypical symptoms than in previous decades [12]. Including in the model, other clinical, laboratory, and instrumental characteristics could improve prediction accuracy; however, adding variables may be expensive and time-consuming and also incorrectly reclassify patients with suspected CAD. Using publicly available dataset, it has been recently reported that ML algorithms have high accuracy to detect the presence of CAD [13]. Yet, if the application of more complex algorithms on traditional risk factor may optimize the estimation of pretest probability of CAD, it remains to be defined. In the present study, we aimed to investigate the potential of different ML techniques for the evaluation of suspected CAD, having as gold standard the presence of stress-induced ischemia by ${ }^{82} \mathrm{Rb}$ positron emission tomography/computed tomography (PET/CT) myocardial perfusion imaging (MPI).
In summary, the main contributions of this work include the following:

(1) A comparison of the value of several ML algorithms in predicting the presence of stress-induced ischemia by noninvasive cardiac imaging

(2) We selected ML algorithms based on their use in the medical field and on the fact that they are representative of different classes of algorithms, such as deterministic, adaptive, and decision tree

The rest of this paper is organized as follows. Section 2 describes the method with detailed information of datasets and ML techniques used. Section 3 describes the results. The discussion is presented in Section 4 followed by the conclusions in Section 5.

\section{Materials and Methods}

2.1. Study Design and Eligibility. Our cohort included a total of 2503 consecutive patients, who underwent cardiac ${ }^{82} \mathrm{Rb}$ $\mathrm{PET} / \mathrm{CT}$ for suspected CAD as part of their diagnostic program between June 2010 and October 2019. Patients with known CAD and patients with acute coronary syndrome were excluded. A patient was considered to have known $\mathrm{CAD}$ at the time of imaging based on a provided history of previously diagnosed atherosclerotic coronary disease, history of myocardial infarction (chest pain or equivalent symptom complex, positive cardiac biomarkers, or typical electrocardiographic changes), history of percutaneous coronary intervention, or history of coronary artery bypass grafting. For patients undergoing more than one PET/CT study, only the earliest procedure was considered. All patients were part of ongoing prospective dedicated database [14]. This study complies with the Declaration of Helsinki. The review committee of our institution approved this study (Ethics Committee, University Federico II, protocol number 110/ 17), and all patients gave informed consent.

2.2. Clinical Definitions. Chest pain was classified according to the American College of Cardiology/American Heart Association 2002 guideline update on exercise testing [15]. Patients were considered as having diabetes if they were receiving treatment with oral hypoglycemic drugs or insulin. A family history of premature CAD was defined as a diagnosis of CAD in a first-degree relative prior to or at 55 years of age. Hypertension was defined as a blood pressure $>140 / 90$ $\mathrm{mm} \mathrm{Hg}$ or use of antihypertensive medication. Hyperlipidemia was defined as total cholesterol level $>6.2 \mathrm{mmol} / \mathrm{L}$ or treatment with cholesterol lowering medication. Smoking history was defined as prior or current tobacco use. Body mass index (BMI) was dichotomized with cut-off to 30 , according to obesity definition.

2.3. PET/CT Imaging. As a routine preparation for ${ }^{82} \mathrm{Rb}$ cardiac PET/CT, patients were asked to discontinue taking methylxanthine containing foods or beverages for 24 hours. Scans were acquired using a Biograph mCT 64-slice scanner (Siemens Healthcare). Rest and stress cardiac PET/CT 


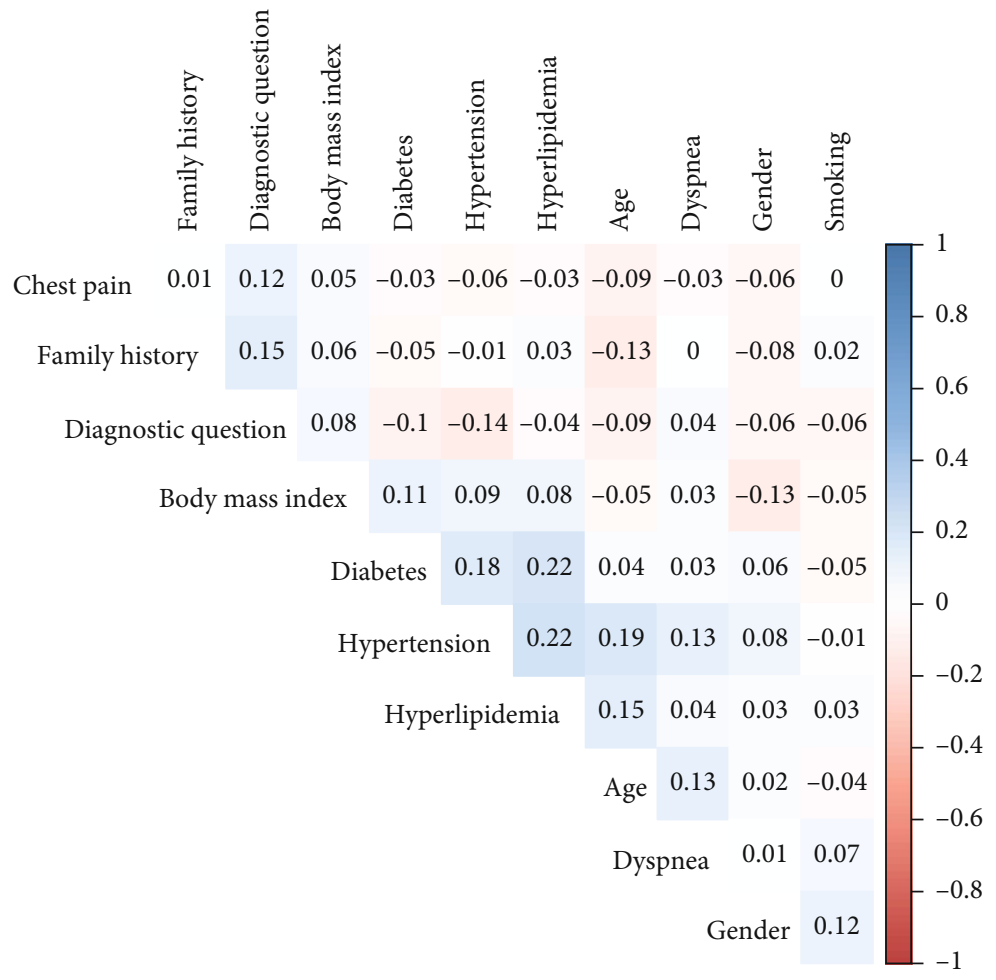

FIGURE 1: Correlation matrix of the features used. The matrix elements are displayed in hierarchical clustering order. The numbers indicate the Spearman $\rho$ coefficient between two features.
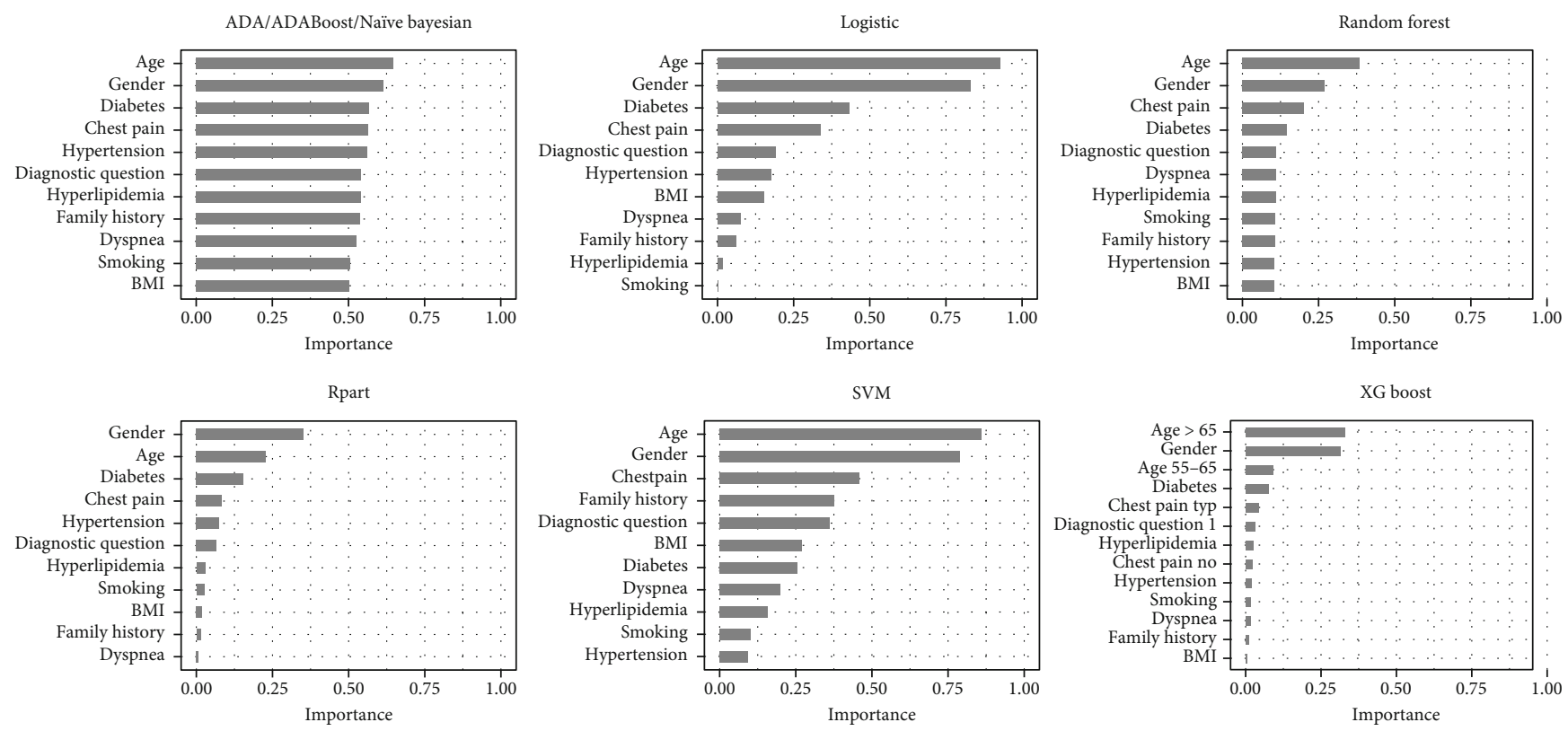

FIGURE 2: Importance of the features for each ML algorithm. ADA, AdaBoost, and Naïve Bayesian features importance were grouped into a single bar plot as the values for the two adaptive algorithms turned out to be equals, and Naïve Bayesian values differed with them by less than $5 \%$.

images were acquired as follows: scout CT was performed to check patient position, and low-dose CT $(0.4 \mathrm{mSv} ; 120 \mathrm{kVp}$; effective tube current, $26 \mathrm{~mA}$ [11-mAs quality reference]; 3.3 seconds) was performed for attenuation correction, during normal breathing before and after PET acquisitions. For both rest and stress images, $1110 \mathrm{MBq}$ of ${ }^{82} \mathrm{Rb}$ was injected intravenously with a 7-minute list-mode PET acquisition. Dynamic PET acquisition was started at rest followed by adenosine pharmacologic stress $\left(140 \mu \mathrm{g} \times \mathrm{kg}^{-1} \times \mathrm{min}^{-1}\right.$ for 4.5 minutes, with tracer administration between 2 and 2.5 
TABLE 2: Values used for tuning of parameters for each ML technique.

\begin{tabular}{|c|c|c|c|}
\hline & Parameter & Parameter space & Chosen value \\
\hline \multirow{3}{*}{$\mathrm{ADA}$} & Number of trees & $10,25,50,100,200$ & 25 \\
\hline & Max tree depth & $5,10,20,50$ & 10 \\
\hline & Learning rate & $0.001,0.005,0.01,0.05,0.1,0.5$ & 0.01 \\
\hline \multirow{2}{*}{ AdaBoost } & Number of trees & $10,25,50,100,200$ & 50 \\
\hline & Method & AdaBoost.M1, real AdaBoost & AdaBoost.M1 \\
\hline Logistic & Family & Binomial & Binomial \\
\hline \multirow{3}{*}{ Naïve Bayes } & Laplace correction & $0,0.5,1.0$ & 0 \\
\hline & Distribution type (kernel) & True, false & False \\
\hline & Bandwidth adjustment & $0.01,0.05,0.1,0.5,1.0$ & 0.1 \\
\hline Random Forest & Number of randomly selected predictors & $3,5,10,20$ & 10 \\
\hline \multirow{4}{*}{ Rpart } & Minimum number of observations in a node & $10,15,30$ & 15 \\
\hline & Minimum number of observations in any leaf node & $3,5,10$ & 5 \\
\hline & Max tree depth & $3,5,10,20$ & 10 \\
\hline & Complexity parameter of the tree & $0.0001,0.001,0.01,0.1$ & 0.001 \\
\hline \multirow{3}{*}{ SVM } & Kernel & Linear, radial, sigmoid & Sigmoid \\
\hline & Parameter needed for sigmoid & $0.05,0.1,0.25,0.5$ & 0.1 \\
\hline & Cost & $0.5,1,2,5$ & 1 \\
\hline \multirow{4}{*}{ XGBoost } & Number of trees & $25,50,100,200$ & 100 \\
\hline & Max tree depth & $5,10,20$ & 10 \\
\hline & Learning rate & $0.001,0.005,0.01,0.05,0.1,0.5$ & 0.01 \\
\hline & Subsamples & $0.5,0.75,1$ & 1 \\
\hline
\end{tabular}

TABLe 3: Metrics obtained from the ML techniques, evaluated on training/test and validation approaches.

\begin{tabular}{|c|c|c|c|c|c|c|c|c|}
\hline & \multicolumn{4}{|c|}{ Training/test $(n=2003)$} & \multicolumn{4}{|c|}{ Validation $(n=500)$} \\
\hline & $\begin{array}{c}\text { Accuracy } \\
(\%)\end{array}$ & $\begin{array}{c}\text { Sensitivity } \\
(\%)\end{array}$ & $\begin{array}{c}\text { Specificity } \\
(\%)\end{array}$ & $\begin{array}{c}\text { AUROC } \\
(\%)\end{array}$ & $\begin{array}{c}\text { Accuracy } \\
(\%)\end{array}$ & $\begin{array}{c}\text { Sensitivity } \\
(\%)\end{array}$ & $\begin{array}{c}\text { Specificity } \\
(\%)\end{array}$ & $\begin{array}{c}\text { AUROC } \\
(\%)\end{array}$ \\
\hline $\mathrm{ADA}$ & 88 & 48 & 97 & 90 & 76 & 26 & 89 & 68 \\
\hline AdaBoost & 89 & 67 & 95 & 95 & 71 & 23 & 87 & 66 \\
\hline Logistic & 80 & 5 & 98 & 72 & 80 & 7 & 98 & 75 \\
\hline Naïve Bayes & 77 & 23 & 91 & 70 & 80 & 27 & 92 & 73 \\
\hline $\begin{array}{l}\text { Random } \\
\text { Forest }\end{array}$ & 89 & 51 & 98 & 93 & 75 & 21 & 89 & 65 \\
\hline Rpart & 82 & 27 & 96 & 75 & 76 & 17 & 91 & 70 \\
\hline SVM & 72 & 13 & 87 & 61 & 77 & 21 & 91 & 65 \\
\hline XGBoost & 83 & 27 & 97 & 83 & 77 & 18 & 92 & 69 \\
\hline
\end{tabular}

minutes). Rest and stress dynamic images were reconstructed into 26-time frames $(12 \times 5$ seconds, $6 \times 10$ seconds, $4 \times 20$ seconds, and $4 \times 40$ seconds; total, 6 minutes) using the vendor standard ordered subsets expectation maximization $3 \mathrm{D}$ reconstruction (2 iterations, 24 subsets) with $6.5 \mathrm{~mm}$ Gaussian postprocessing filter. In addition, the images were corrected for attenuation using the low-dose CT. The heart rate, systemic blood pressure, and 12-lead ECG were recorded at baseline and throughout the infusion of adenosine. An automated software program (e-soft, 2.5, QGS/QPS, Cedars-Sinai Medical Center, Los Angeles, CA) was used to calculate the scores (summed stress score, summed rest score, and summed difference score) incorporating both the extent and severity of perfusion defects, using the standardized segmentation of 17 myocardial regions $[16,17]$. A summed difference score $\geq 2$ was considered ischemic.

2.4. Statistical Analysis. Statistical analysis was performed using the $R$ software, version 3.6.2 (The $R$ Foundation for Statistical Software, Vienna, Austria). Two-sided $P$ values $<0.05$ were considered statistically significant. The dataset 


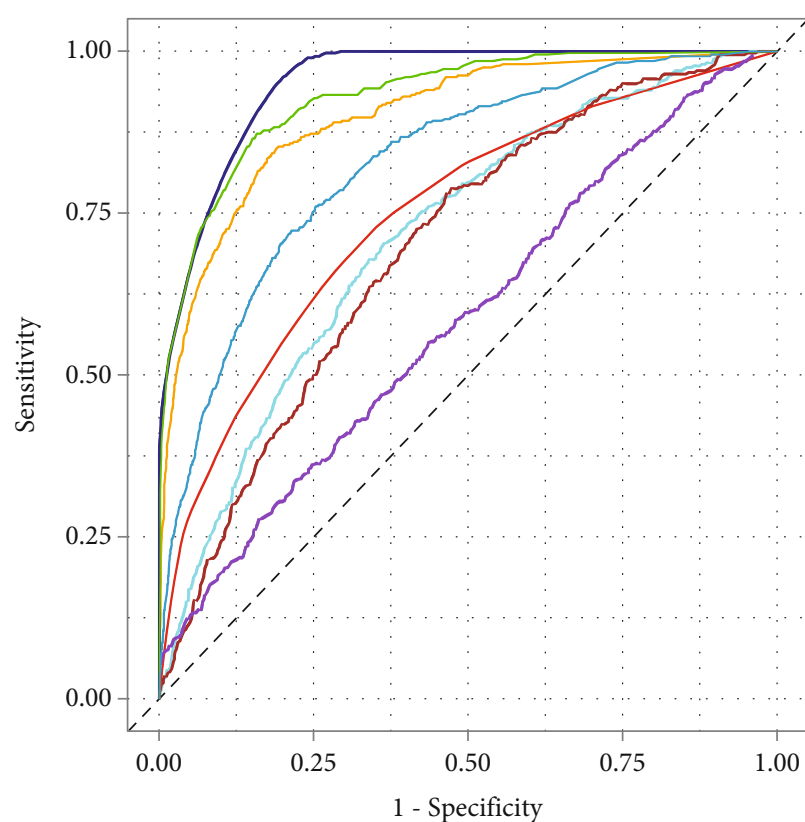

ML (AUROC)

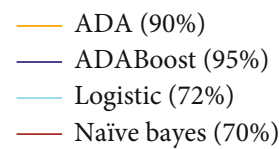

(a)

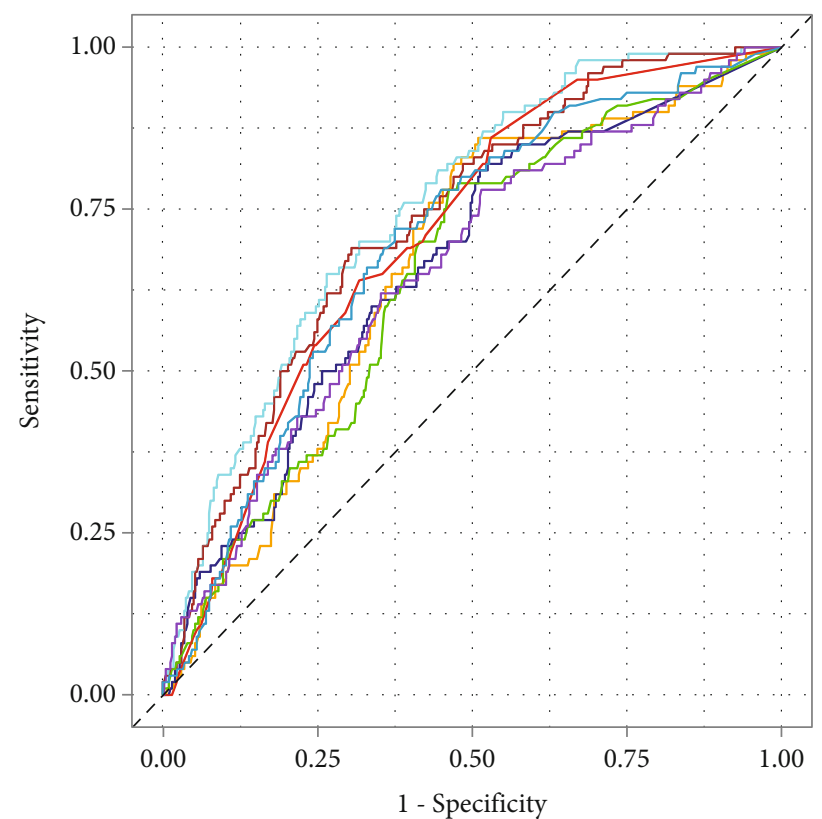

ML (AUROC)
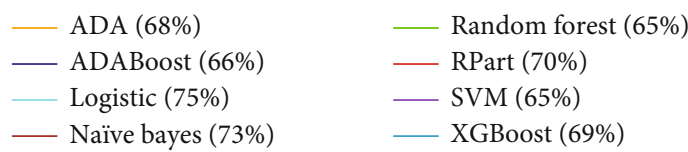

(b)

FIGURE 3: Comparison among the ROC curves of the eight ML techniques considered. The ML performances are reported separately for the training/test approach (a) and validation approach (b). Parenthesis are reported the AUROC values.

consisted of 11 features, of which 10 demographic or clinical variables (age, gender, BMI, typical or atypical chest pain, diabetes mellitus, dyspnea, family history, hypertension, hyperlipidemia, smoking), and the diagnostic question with two categories: diagnostic or presurgery evaluation. Age and BMI continuous variables were categorized $(<55,55-$ $65,>65$ years, and $\mathrm{BMI}<30$ ); then, all data were expressed as percentages. Differences between groups were analyzed by $\chi^{2}$ test. The correlation among features was tested by Spearman $\rho$ coefficient, embedded in the corrplot package. This nonparametric test is appropriate to evaluate the correlation between categorical variables and to find redundant features. Data in input to ML algorithms were normalized. Sensitivity, specificity, and accuracy were computed using the confusionMatrix function embedded in the caret package. Sensitivity evaluated how good a ML is for detecting the positive patients (i.e., ischemic according to MPI results), and its numeric value was obtained by ratio between the number of patients correctly assessed as positive by ML and the number of positive patients. Specificity evaluated the negative patients (i.e., normal according to the MPI results), and it was calculated by ratio between the number of patients correctly assessed as negative by ML and the number of negative patients. Accuracy measured how correctly a ML identified and excluded a given condition, and it was obtained from the ratio between the number of patients correctly assessed by ML and the total number of patients. Receiver operating characteristic curve is a graphic presentation of the relationship between sensitivity and specificity, whereas the area under this curve provides a measurement of the correct evaluation of ML with respect a random classifier. The areas under the receiver operating characteristic (AUROC) curves were computed by the $p R O C$ package.

2.5. ML Techniques. For the comparison presented in this study, we selected supervised ML algorithms, appropriate to categorical data for a binary response. We used the algorithms developed in $R$. ADA is a classification tree based on adaptive algorithms, used to fit a variety stochastic boosting. This algorithm can be used in conjunction with other types of learning procedures to improve performance. The output of these procedures, called weak learners, is combined into a weighted sum that represents the final output of the boosted classifier [18]. AdaBoost is a classifier similar to ADA, differing from this for the AdaBoost.M1 algorithm implemented by Freund and Schapire [19]. Logistic algorithm used in this study is a part of generalized linear models [20]. This classifier was chosen as a reference because adopted in clinical statistical analysis, with categorical or numerical data and dichotomous response. The equation assumed a linear relationship between the predictor variables $x_{i}$ and the log odds 


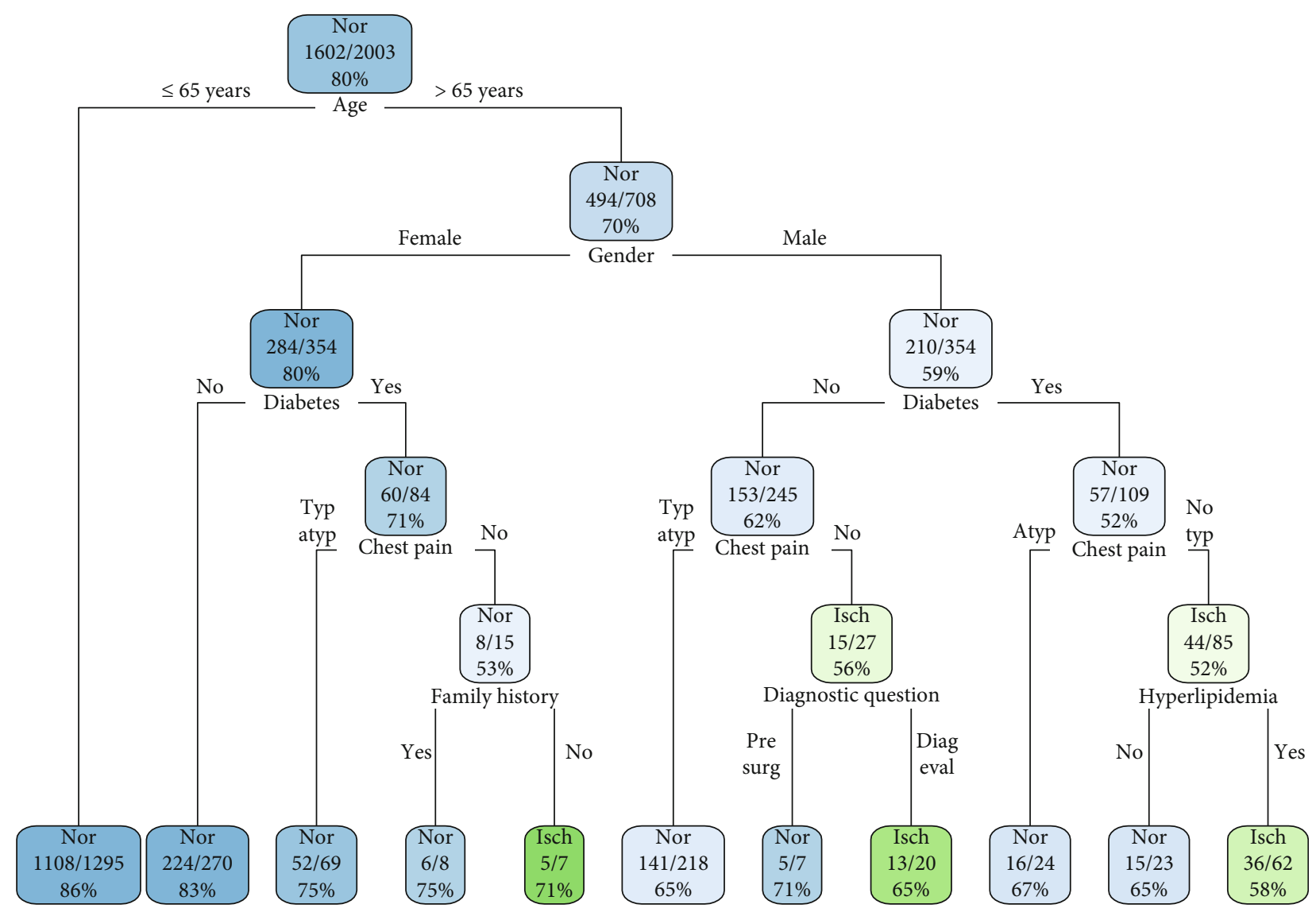

FIGURE 4: Decision tree obtained by rpart algorithm. Each node or leaf is reported the prevalence concerning MPI outcome (nor: normal; isch: ischemic), the ratio between the number of prevalent and total patients, and the relative percentage.

(in term of probability $p$ ) of the event, as follows:

$$
\log \frac{p}{1-p}=\beta_{0}+\sum_{i=1}^{n} \beta_{i} x_{i} .
$$

Then, the $\beta$ coefficients are determinates, with $\beta_{0}$ representing the particular case with all variables equal to zero. The Naïve Bayes is a probabilistic classifier based on the Bayes' theorem. This algorithm requires a strong (naïve) independence assumption between the features [21]. Random Forest is an algorithm based on an ensemble learning method for classification and regression that operate by constructing a multitude of decision trees at training time. The procedure returns as output the class that is the mode of the classes (for classification) or average prediction (for regression) of the individual trees [22]. Rpart is a decision tree algorithm that works by splitting in two parts the dataset recursively. For each step, the split is obtained considering the feature that results in the largest possible reduction in heterogeneity of the outcome variable [23]. Support vector machine (SVM) is an algorithm that constructs hyperplanes in a highdimensional space, which can be used for classification and regression [24]. SVM is a robust prediction method that can efficiently perform nonlinear classifications, by appropriate kernels. XGBoost is a scalable end-to-end tree boost- ing method, based on a sparsity-aware algorithm for sparse data and weighted quantile sketch for approximate tree learning [25].

2.6. Approaches Used for the ML Evaluation. To testing the ML performances, the data were split randomly into two parts: training/test (80\%) and validation (20\%). For the training/test of data, we applied a 5-fold cross-validation method, repeated 2 times. With this subset, we performed the tuning of free parameters for each algorithm. For both training/test and validation, we computed accuracy, sensitivity, specificity, and AUROC.

2.7. Hardware and Software Characteristics. For this study, we used a common personal computer equipped with a 2.2 GHz Intel i3-2330 quad-core processor, $8 \mathrm{~GB}$ of RAM, and a $0.5 \mathrm{~TB}$ SSD. The operating system was a Windows 10 , whereas the scripts in $R$ programming code were obtained developing inhouse software.

\section{Results}

Demographic and clinical characteristics of study population according to normal or ischemic MPI response are summarized in Table 1. All features, except BMI and smoking, were statistically significant to $\chi^{2}$ test. 
Figure 1 shows the Spearman correlation coefficients matrix of features. All the found absolute values were $<0.25$, highlighting only weak correlations among features. The cluster with higher correlation among features was obtained by diabetes, hypertension, and hyperlipidemia $(\rho=0.22)$. The very low correlation values demonstrated the absence of redundant features.

Figure 2 reports the feature importance for each algorithm. We observed the same feature importance values for ADA and AdaBoost algorithms, whereas small differences $(<5 \%)$ were found between these procedures and the Naïve Bayes ML. Therefore, we reported a unique bar plot for these three algorithms. In general, the most important features were age and gender, followed from diabetes or chest pain. We also observed relevant differences among features importance of most of ML algorithms, except for the two adaptive and Naïve Bayesian algorithms. In fact, for these three algorithms, the importance values were comprised between 0.50 and 0.65 , whereas for the logistic algorithm, we obtained larger interval of values from 0.001 to 0.93 .

Table 2 summarizes the space parameters and the value chosen for the tuning of ML. Parameters were tested using a 5 -fold cross-validation, repeated 2 times, targeted to maximize the C-index. Among all tested setting for each algorithm, we chose the combination with higher sensitivity to balance the result performances.

Table 3 shows the C-statistics results of the ML algorithms, for training/test and validation approaches. In general, the performances in training/test approach were better than of the validation approach. Due to unbalanced dataset, specificity resulted greater than sensitivity. For all metrics, the best performance in training/test was observed for AdaBoost ML. The Naïve Bayes ML resulted to be more efficient in validation approach. ML based on traditional logistic algorithm showed a low sensitivity and similar performance for the training/test and validation approaches. Figure 3 shows a graphical comparison among the ROC curves of the $\mathrm{ML}$ algorithms, for both training/test and validation approaches.

Figure 4 shows the tree generated from the rpart algorithm. To make the decision tree easier to read, the max depth was fixed to 5 . The first spit was on age and for younger patients ( $\leq 65$ years), without any node until the terminal leaf, where a prevalence of normal MPI of $86 \%$ was observed. For older patients ( $>65$ years), the algorithm calculated the gender node, with a percentage of normal MPI of $70 \%$. The split in this node, related to the female gender, was followed by diabetes, chest pain, and family history of CAD.

\section{Discussion}

At best of our knowledge, this is the first study comparing the value of several ML algorithms in predicting the presence of stress-induced ischemia by ${ }^{82} \mathrm{Rb}$ PET/CT cardiac imaging. We selected eight ML algorithms based on their clinical use and on the fact that they are representative of different classes of algorithms, such as deterministic (e.g., SVM), adaptive (e.g., ADA), and decision tree (e.g., rpart).
The results indicate that by adaptive (ADA and AdaBoost) and Random Forest algorithms, AUROC curve was $\geq 90 \%$ in training/test phase.

As input features for the ML algorithms, we considered demographic data and traditional cardiac risk factors. No significant correlations were detectable between variables, a necessary condition for features selection in ML techniques and for data processing. The feature importance is an important step for ML techniques. In our study aside from demographic characteristics, diabetes and chest pain resulted to be the most useful features for predicting stress-induced ischemia by PET/CT. This result confirms another study based on SPECT, where the feature importance, obtained by logistic regression, was the following: gender, age, and chest pain [26]. Noteworthy, features (BMI and smoking) showing not significant $\chi^{2}$ statistic resulted relevant at ML analysis. Indeed, ML algorithms may capture the subtle value of features apparently not significant at conventional analysis.

The ML algorithms showed a variable accuracy (72\%$89 \%$ ) by training/test phase, with low sensitivity and high specificity. This latter finding probably reflects the unbalanced dataset between normal and abnormal MPI and is in agreement with the observation that, in the contemporary pretest probability of CAD, noninvasive imaging tests have greater ruling out that ruling in capabilities [12]. Also, the AUROC values were very wide (61\%-95\%), with better performances for ADA, AdaBoost, and Random Forest. By these ML algorithms, we obtained the greater values of sensitivity. However, these better performances were lower in the validation set, probably due to the ensemble of weakly solutions and a high number of decision trees elaborated during the training/test phase for each of the three ML algorithms. For XGBoost, we observed a similar performance to these three algorithms, but a lower sensitivity. The Naïve Bayes and SVM resulted to have more generalized performances by the two approaches, with lightly better results by validation phase. The logistic and rpart algorithms showed similar metric values for the training/test and validation approaches.

The logistic technique, taken as a reference, did not result particularly performant with respect to the other ML algorithms. In particular, the value of sensitivity was the lowest, probably explainable with the unbalanced dataset. However, the AUROC resulted higher with respect to a similar study $(A U R O C=64 \%)$ based on clinical risk factors, single-photon emission computed tomography imaging, and logistic regression [10].

As an example of a tool for decision-making, we reported the tree obtained by rpart. From a graphic point of view, it is immediate to verify the effect of age and gender on the construction of the decision tree. For younger patients, there is a prevalence of normal MPI, without further ramifications. Otherwise, a gender split is observed, followed in both cases by the split of diabetes and chest pain, with a larger complexity for the male gender.

Previous studies used ML algorithms in cardiology [27], but at the best of our knowledge, no study evaluated this approach to estimate the pretest probability of an ischemic response to PET/CT. In a study based, an XGBoost $\mathrm{ML}$ 
was developed in a large series of symptomatic patients to predict pretest probability of obstructive CAD on coronary computed tomography angiography. The $\mathrm{ML}$ model had significantly higher discrimination (AUROC $=81 \%$ ), as compared to traditional models, with a good sensitivity (91.9\%) but a low (38.8\%) specificity. This study was used a 10-fold cross-validation approach but and no independent validation dataset [28]. In another study [29], a SVM algorithm was used to determine the diagnostic value of joint PET myocardial perfusion and metabolic imaging for predicting obstructive coronary artery disease in symptomatic patients with available coronary angiography. The study included only 88 patients, most of them with known CAD. The joint PET evaluation improves had a good performance (AUROC $=86 \%$ ), and the SVM algorithm outperformed the other methods evaluated. In a study [30], including a total of 16,120 patients, ML improved one-year risk discrimination in predicting durable left ventricular assist devices as compared to logistic regression (C-index $71 \%$ vs. $69 \%, P<0.001$ ); however, calibration metrics were comparable. Globally, these studies confirm limited value of current clinical models to accurately predict the presence of myocardial ischemia at stress MPI [31].

\section{Conclusions}

The results of this study performed in a large series of patients with suspected CAD demonstrate that the classification based on demographic and cardiovascular risk factors has a limited value in validation phase for predicting an ischemic response by ${ }^{82} \mathrm{Rb}$ PET/CT in patients with suspected CAD. We selected eight $\mathrm{ML}$ algorithms that are implemented by different software packages and can be used by other researchers on their MPI data. Other ML algorithms, such as monarch butterfly optimization [32], earthworm optimization algorithm [33], elephant farming optimization [34, 35], moth search algorithm [36], slime mould algorithm [37], and Harris hawks optimization [38], can also be used to predict stress-induced ischemia by MPI and should be tested in future studies. In conclusion, the role of other clinical and instrumental characteristics, as well as developing and perfecting more complex algorithms to improve the prediction of stress-induced ischemia by MPI, remains a work in progress.

\section{Data Availability}

The data used in this study are available from the corresponding author on a reasonable request.

\section{Ethical Approval}

This study was approved by the Ethics Committee of the University of Naples Federico II, and written informed consent was obtained from each participant.

\section{Conflicts of Interest}

The authors declare that there is no conflict of interest regarding the publication of this paper.

\section{Authors' Contributions}

Rosario Megna, Mario Petretta, and Alberto Cuocolo conceptualized the study and drafted the manuscript. Rosario Megna, Roberta Assante, Emilia Zampella, Carmela Nappi, Valeria Gaudieri, Teresa Mannarino, Adriana D’Antonio, Roberta Green, Valeria Cantoni, Parthiban Arumugam, and Wanda Acampa collected and analyzed the data. All the authors revised and commented on the paper and approved the final version of the manuscript.

\section{Acknowledgments}

We would like to express our gratitude to the staff of the Division of Nuclear Medicine for their excellent technical support.

\section{References}

[1] P. Chen, P. C. Chen, Y. Liu, and L. Peng, "How to develop machine learning models for healthcare," Nature Materials, vol. 18, no. 5, pp. 410-414, 2019.

[2] R. C. Deo, "Machine learning in medicine," Circulation, vol. 132, no. 20, pp. 1920-1930, 2015.

[3] A. Rajkomar, J. Dean, I. Kohane, and I. Kohane, "Machine learning in medicine," New England Journal of Medicine, vol. 380, no. 14, pp. 1347-1358, 2019.

[4] R. Megna, A. Cuocolo, and M. Petretta, "Applications of machine learning in medicine," Biomedical Journal of Scientific \& Technical Research, vol. 20, no. 5, pp. 15350-15352, 2019.

[5] L. M. Stevens, B. J. Mortazavi, R. C. Deo, L. Curtis, and D. P. Kao, "Recommendations for reporting machine learning analyses in clinical research," Circulation: Cardiovascular Quality and Outcomes, vol. 13, no. 10, article e006556, 2020.

[6] C. Ricciardi, R. Cuocolo, R. Megna, M. Cesarelli, and M. Petretta, "Machine learning analysis: general features, requirements and cardiovascular applications," Minerva Cardiology and Angiology, 2021.

[7] G. A. Diamond and J. S. Forrester, "Analysis of probability as an aid in the clinical diagnosis of coronary-artery disease," New England Journal of Medicine, vol. 300, no. 24, pp. 13501358, 1979.

[8] T. S. Genders, E. W. Steyerberg, M. G. Hunink et al., "Prediction model to estimate presence of coronary artery disease: retrospective pooled analysis of existing cohorts," BMJ, vol. 344, no. jun12 1, article e3485, 2012.

[9] J. Reeh, C. B. Therming, M. Heitmann et al., "Prediction of obstructive coronary artery disease and prognosis in patients with suspected stable angina," European Heart Journal, vol. 40, no. 18, pp. 1426-1435, 2019.

[10] R. Megna, R. Assante, E. Zampella et al., "Pretest models for predicting abnormal stress single-photon emission computed tomography myocardial perfusion imaging," Journal of Nuclear Cardiology, 2019.

[11] R. Megna, C. Nappi, V. Gaudieri et al., "Diagnostic value of clinical risk scores for predicting normal stress myocardial 
perfusion imaging in subjects without coronary artery calcium," Journal of Nuclear Cardiology, 2020.

[12] L. E. Juarez-Orozco, A. Saraste, D. Capodanno et al., "Impact of a decreasing pre-test probability on the performance of diagnostic tests for coronary artery disease," European Heart Journal - Cardiovascular Imaging, vol. 20, no. 11, pp. 11981207, 2019.

[13] A. Akella and S. Akella, "Machine learning algorithms for predicting coronary artery disease: efforts toward an open source solution," Future Science OA, vol. 7, no. 6, article FSO698, 2021.

[14] R. Megna, M. Petretta, B. Alfano et al., "A new relational database including clinical data and myocardial perfusion imaging findings in coronary artery disease," Current Medical Imaging, vol. 15, no. 7, pp. 661-671, 2019.

[15] Committee Members, R. J. Gibbons, G. J. Balady et al., "ACC/ AHA 2002 guideline update for exercise testing: summary Article," Circulation, vol. 106, no. 14, pp. 1883-1892, 2002.

[16] D. S. Berman, A. Abidov, X. Kang et al., "Prognostic validation of a 17-segment score derived from a 20-segment score for myocardial perfusion SPECT interpretation," Journal of Nuclear Cardiology, vol. 11, no. 4, pp. 414-423, 2004.

[17] H. J. Verberne, W. Acampa, C. Anagnostopoulos et al., "EANM procedural guidelines for radionuclide myocardial perfusion imaging with SPECT and SPECT/CT: 2015 revision," European Journal of Nuclear Medicine and Molecular Imaging, vol. 42, no. 12, pp. 1929-1940, 2015.

[18] J. Friedman, T. Hastie, and R. Tibshirani, "Additive logistic regression: a statistical view of boosting (With discussion and a rejoinder by the authors)," The Annals of Statistics, vol. 28, no. 2, pp. 337-407, 2000.

[19] Y. Freund and R. E. Schapire, "Experiments with a new boosting algorithm," in Proceedings of the Thirteenth International Conference on Machine Learning, pp. 148-156, Morgan Kaufmann, 1996.

[20] P. McCullagh and J. A. Nelder, Generalized Linear Models, Chapman \& Hall/CRC, London, UK, 2nd ed edition, 1989.

[21] A. McCallum and N. Kamal, "A comparison of event models for Naive Bayes text classification," in AAAI-98 workshop on learning for text categorization, Madison, Wisconsin, July 1998.

[22] L. Breiman, "Random forests," Machine Learning, vol. 45, no. 1, pp. 5-32, 2001.

[23] L. Breiman, J. H. Friedman, R. A. Olshen, and C. J. Stone, Classification and Regression Trees, Routledge, Wadsworth, 1984.

[24] C. Cortes and V. Vapnik, "Support-vector networks," Machine Learning, vol. 20, no. 3, pp. 273-297, 1995.

[25] T. Chen and C. Guestrin, "XGBoost: a scalable tree boosting system," in 22nd ACM SIGKDD International Conference on Knowledge Discovery and Data Mining, pp. 785-794, San Francisco, California, USA, 2016.

[26] R. Megna, E. Zampella, R. Assante et al., “Temporal trends of abnormal myocardial perfusion imaging in a cohort of Italian subjects: relation with cardiovascular risk factors," Journal of Nuclear Cardiology, vol. 27, no. 6, pp. 2167-2177, 2020.

[27] R. Cuocolo, T. Perillo, E. De Rosa, L. Ugga, and M. Petretta, "Current applications of big data and machine learning in cardiology," Journal of Geriatric Cardiology, vol. 16, no. 8, pp. 601-607, 2019.

[28] Z. H. Hou, B. Lu, Z. N. Li et al., "Machine learning for pretest probability of obstructive coronary stenosis in symptomatic patients," JACC Cardiovascular Imaging, vol. 12, no. 12, pp. 2584-2586, 2019.

[29] F. Wang, W. Xu, W. Lv et al., "Evaluation of the diagnostic value of joint PET myocardial perfusion and metabolic imaging for vascular stenosis in patients with obstructive coronary artery disease," Journal of Nuclear Cardiology, 2020.

[30] A. Kilic, D. Dochtermann, R. Padman, J. K. Miller, and A. Dubrawski, "Using machine learning to improve risk prediction in durable left ventricular assist devices," PLoS One, vol. 16, no. 3, article e0247866, 2021.

[31] T. S. Dunn 2nd and F. G. Hage, "Stress myocardial perfusion imaging: can we tell the results without doing the test?," Journal of Nuclear Cardiology, 2020.

[32] Y. Feng, S. Deb, G. G. Wang, and A. H. Alavi, "Monarch butterfly optimization: a comprehensive review," Expert Systems with Applications, vol. 168, article 114418, 2021.

[33] G. G. Wang, S. Deb, and L. D. S. Coelho, "Earthworm optimization algorithm: a bio-inspired metaheuristic algorithm for global optimization problems," International Journal of BioInspired Computation, vol. 1, no. 1, p. 1, 2015.

[34] G. G. Wang, S. Deb, X. Z. Gao, and L. D. S. Coelho, "A new metaheuristic optimisation algorithm motivated by elephant herding behaviour," International Journal of Bio-Inspired Computation, vol. 8, no. 6, p. 394, 2016.

[35] M. A. Elhosseini, R. A. El Sehiemy, Y. I. Rashwan, and X. Z. Gao, "On the performance improvement of elephant herding optimization algorithm," Knowledge-Based Systems, vol. 166, pp. 58-70, 2019.

[36] G. G. Wang, "Moth search algorithm: a bio-inspired metaheuristic algorithm for global optimization problems," Memetic Computing, vol. 10, no. 2, pp. 151-164, 2018.

[37] S. Li, H. Chen, M. Wang, A. A. Heidari, and S. Mirjalili, "Slime mould algorithm: a new method for stochastic optimization," Future Generation Computer Systems., vol. 111, pp. 300-323, 2020.

[38] A. A. Heidari, S. Mirjalili, H. Faris, I. Aljarah, M. Mafarja, and H. Chen, "Harris hawks optimization: algorithm and applications," Future Generation Computer Systems, vol. 97, pp. 849-872, 2019. 